\title{
Singlet molecular oxygen by direct excitation
}

\author{
Steffen Jockusch, ${ }^{a}$ Nicholas J. Turro, ${ }^{a}$ Elizabeth K. Thompson, ${ }^{b}$ Martin Gouterman, ${ }^{b}$ James B. Callis ${ }^{b}$ and \\ Gamal E. Khalil $* b$
}

Received 17th September 2007, Accepted 14th November 2007

First published as an Advance Article on the web 30th November 2007

DOI: $10.1039 / b 714286 b$

Direct excitation at $1064 \mathrm{~nm}$ and detection of singlet molecular oxygen at $1270 \mathrm{~nm}$ is made possible by the availability of powerful YAG-lasers and sensitive NIR photomultipliers. Singlet oxygen was generated in condensed phase at $77 \mathrm{~K}$ by direct excitation at $1064 \mathrm{~nm}$ (without the use of sensitizers). Several luminescing species were observed by time resolved luminescence spectroscopy and luminescence lifetime measurements, including the single molecule ${ }^{1} \Delta_{\mathrm{g}}$ and ${ }^{1} \Sigma_{\mathrm{g}}{ }^{+}$states as well as luminescence from the $\left[{ }^{1} \Delta_{\mathrm{g}}\right]_{2}$ simultaneous transition. As an application we propose a novel method for obtaining quantitative non-intrusive mapping of the 2-D oxygen concentrations and pressure at cryogenic temperatures, which is of importance in aircraft design for high altitudes.

\section{Introduction}

Molecular oxygen is a paramagnetic molecule with an even number of electrons. The prediction by Mulliken ${ }^{1,2}$ that the ground electronic state is ${ }^{3} \Sigma_{\mathrm{g}}{ }^{-}$was one of the early triumphs of molecular orbital theory. The lowest singlet excited states ${ }^{1} \Delta_{\mathrm{g}}$ and ${ }^{1} \Sigma_{\mathrm{g}}{ }^{+}$lie at $7882 \mathrm{~cm}^{-1}$ and $13121 \mathrm{~cm}^{-1}$, respectively, above the ground state. In the free unperturbed molecule, the transitions from the ground state to ${ }^{1} \Delta_{\mathrm{g}}$ and ${ }^{1} \Sigma_{\mathrm{g}}{ }^{+}$are electric dipole and spin forbidden and gain intensity mainly through magnetic dipole moment. The review by Schweitzer and Schmidt provides a comprehensive discussion of the physical process of singlet oxygen generation and deactivation. ${ }^{3}$ They report calculated radiative lifetimes of 72 minutes for ${ }^{1} \Delta_{\mathrm{g}}$ and 11 seconds for ${ }^{1} \Sigma_{\mathrm{g}}{ }^{+}$. However, at higher oxygen concentrations and in the presence of other molecular species these transition rates rise up to $\sim 10^{4}$ times due to intermolecular perturbations, which enhance the radiative processes. ${ }^{4,5}$ The observed lifetimes for ${ }^{1} \Delta_{\mathrm{g}}$ range from milliseconds in gaseous phase to microseconds in aqueous media. In contrast the lifetime of ${ }^{1} \Sigma_{\mathrm{g}}{ }^{+}$is much shorter since the excited state quickly decays into ${ }^{1} \Delta_{\mathrm{g}}$. The effect of concentration, solvents and environments on the molecular oxygen transitions has been extensively studied. ${ }^{6,7}$

Surprisingly for a homogeneous diatomic molecule, in the condensed phase or high gas pressure the absorption spectrum of molecular oxygen spans the UV-NIR spectral range. In addition to the expected single molecule bands for ${ }^{1} \Delta_{\mathrm{g}} \leftarrow{ }^{3} \Sigma_{\mathrm{g}}{ }^{-}$and ${ }^{1} \Sigma_{\mathrm{g}}{ }^{+} \leftarrow{ }^{3} \Sigma_{\mathrm{g}}{ }^{-}$ transitions, one observes additional absorption bands that arise from dimer $\left(\mathrm{O}_{2}\right)_{2}$ configurations and correspond to various pairs of single molecule transitions as shown in Fig. 1. The intensities of these bands have quadratic dependency on the oxygen concentration and are responsible for the blue color of liquid oxygen. This phenomenon is interpreted as an up-conversion process of two oxygen molecules being excited simultaneously by one photon to a virtual state not common to either molecule. ${ }^{8}$ The energy of the up-conversion transition is equal to the sum of the transition energies. This cooperative process was rediscovered in emission by

${ }^{a}$ Department of Chemistry, Columbia University, New York, NY, 10027

${ }^{b}$ Department of Chemistry, University of Washington, Seattle, WA, 98195. E-mail: gamalk@u.washington.edu

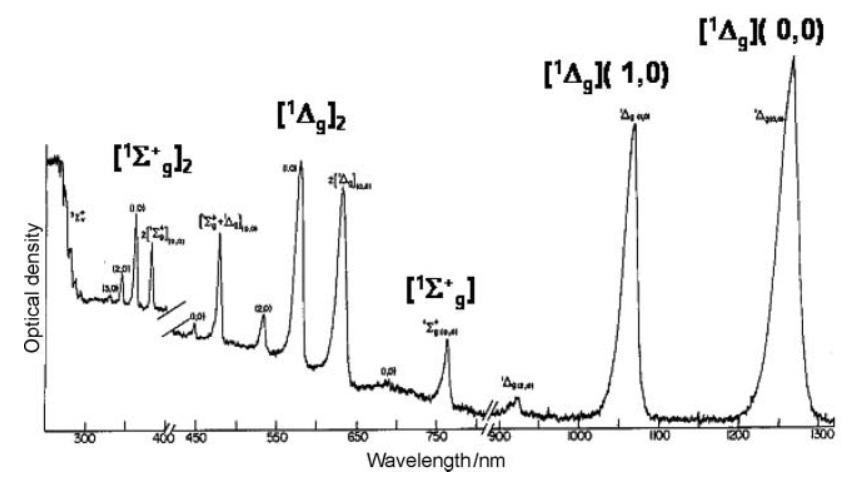

Fig. 1 Absorption spectrum of liquid oxygen (optical cell length is 3.5 $\mathrm{cm}$ ). Scale is adjusted for plotting purposes. After G. Khalil, PhD thesis, Florida State University, 1978.

Khan and $\mathrm{Kasha}^{9}$ by measuring the chemiluminescence reaction (eqn (1)):

$$
\mathrm{H}_{2} \mathrm{O}_{2}+\mathrm{OCl}^{-} \rightarrow{ }^{1} \mathrm{O}_{2}\left({ }^{1} \Sigma_{\mathrm{g}}{ }^{+} \text {and }{ }^{1} \Delta_{\mathrm{g}}\right)+\mathrm{Cl}^{-}+\mathrm{H}_{2} \mathrm{O} .
$$

These workers observed the following transition bands:

$$
\begin{gathered}
{ }^{3}\left[\left({ }^{1} \Sigma_{\mathrm{g}}{ }^{+}\right)\left({ }^{3} \Sigma_{\mathrm{g}}{ }^{-}\right)\right] \leftrightarrow{ }^{1,3,5}\left[\left({ }^{3} \Sigma_{\mathrm{g}}{ }^{-}\right)\left({ }^{3} \Sigma_{\mathrm{g}}{ }^{-}\right)\right](\text {at } 763 \mathrm{~nm}) \\
{ }^{1}\left[\left({ }^{1} \Delta_{\mathrm{g}}\right)\left({ }^{1} \Delta_{\mathrm{g}}\right)\right] \leftrightarrow{ }^{1,3,5}\left[\left({ }^{3} \Sigma_{\mathrm{g}}{ }^{-}\right)\left({ }^{3} \Sigma_{\mathrm{g}}{ }^{-}\right)\right](\text {at } 633 \mathrm{~nm}, 703 \mathrm{~nm}) \\
{ }^{1}\left[\left({ }^{1} \Sigma_{\mathrm{g}}{ }^{+}\right)\left({ }^{1} \Delta_{\mathrm{g}}\right)\right] \leftrightarrow{ }^{1,3,5}\left[\left({ }^{3} \Sigma_{\mathrm{g}}{ }^{-}\right)\left({ }^{3} \Sigma_{\mathrm{g}}{ }^{-}\right](\text {at } 478 \mathrm{~nm})\right.
\end{gathered}
$$

Transition (2) is a single molecule emission event; transitions (3) and (4) are cooperative emission process of two molecules. Combined spin multiplicity notation was used to illustrate the enhancement of the spin selection rule for molecular pair oxygen states. The Ogryzlo group recorded similar emission spectra from an electrical discharge of gaseous molecular oxygen. ${ }^{10}$ This pioneering work and others have ignited interest in singlet molecular oxygen research and over the last 30 years several conference proceedings on singlet oxygen and its applications have been published..$^{11-14}$

Generating singlet oxygen by a photo-sensitizer is the most efficient process and up to $100 \%{ }^{1} \Delta_{\mathrm{g}}$ generation efficiency has been 
reported. ${ }^{15,16}$ Using a platinum porphyrin sensor film we recently demonstrated a new method to measure oxygen concentration on a 2-D surface. ${ }^{17}$ The method is based on the porphyrin-sensitized $1270 \mathrm{~nm}$ singlet oxygen emission imaged, with an InGaAs camera as a function of surface pressure. Several research groups have previously determined the lifetime of singlet oxygen directly by time resolved spectroscopy. ${ }^{3,18}$

Generating singlet oxygen by direct excitation became efficient and feasible with the arrival of powerful lasers. Skuja and Güttler detected ${ }^{1} \Delta_{\mathrm{g}}$ emission from trapped molecular oxygen gas in $\mathrm{SiO}_{2}$ glass by direct laser photoexcitation. ${ }^{19}$ The Krasnovsky research group recently produced the action spectrum of the photooxygenation of tetracene and 1,3-diphenylisobenzofuran due to direct formation of the singlet oxygen state ${ }^{1} \Delta_{\mathrm{g}}$ by laser excitation. ${ }^{20}$ It was found that the reaction rate is linearly dependent on the laser power and strongly diminished in the presence of singlet oxygen scavengers.

We take advantage of the fortuity that the YAG laser $1064 \mathrm{~nm}$ line is a close match to the molecular oxygen transition $\left[{ }^{1} \Delta_{\mathrm{g}}(v=\right.$ $1)] \leftarrow\left[{ }^{3} \Sigma_{\mathrm{g}}{ }^{-}(v=0)\right]$ absorption band. With the availability of fast sensitive NIR detectors, detection of ${ }^{1} \Delta_{\mathrm{g}}$ emission becomes much more feasible for direct oxygen concentration measurement and potential surface pressure mapping applications. This present work is a spectroscopic study of the singlet molecular oxygen state generated by direct excitation. Emission spectra and lifetime measurements from the single molecule ${ }^{1} \Delta_{\mathrm{g}}$ and ${ }^{1} \Sigma_{\mathrm{g}}{ }^{+}$states, as well as from the $\left[{ }^{1} \Delta_{\mathrm{g}}\right]_{2}$ simultaneous transition are presented for the first time.

\section{Materials and methods}

An empty pyrex NMR tube ( $4 \mathrm{~mm}$ diameter) was placed in a liquid nitrogen filled dewar, which possesses an optically transparent section for spectroscopic measurements. Liquid oxygen or liquid nitrogen-oxygen mixtures were condensed into the NMR tube by flushing the NMR tube with oxygen gas or nitrogen-oxygen gas mixtures prior to the measurements. A temperature of $150 \mathrm{~K}$ was obtained by careful addition of liquid nitrogen into n-pentane. For direct excitation of triplet oxygen (condensed oxygen in the NMR tube at $77 \mathrm{~K}$ ), the pulses from a Spectra Physics NdYAG laser (GCR-150-30) at $1064 \mathrm{~nm}$ (pulse width $10 \mathrm{~ns}$; $\sim 100 \mathrm{~mJ} \mathrm{pulse}^{-1}$ ) were used. The molecular oxygen emissions were collected employing a modified Fluorolog 2 spectrometer (JobinYvon Inc.) in conjunction with Hamamatsu photomultiplier tubes (PMT) (R928 for the visible spectral range and H9170-45 for the NIR spectral range). A glass cut-off filter at $800 \mathrm{~nm}$ was fitted in front of the sample to eliminate scattered light from laser pump flash-lamps. For recording luminescence decay traces at fixed emission wavelength, the photocurrent from the PMT was amplified (SR 560, Stanford Research Systems) and stored in a digital oscilloscope (TDS 360, Tektronix). For recording time resolved luminescence spectra, the amplified photocurrent from the PMT was time resolved using a boxcar integrator (SR 250, Stanford Research Systems). The time-gated signal from the boxcar integrator was digitized with a DM303 M module (Jobin-Yvon Inc.), then stored and synchronized with the monochromator stepping motor using the software supplied by the Fluorolog 2 spectrometer. Scattering interference from the intense laser line was eliminated by integrating over time periods of $15 \mu \mathrm{s}$ of the emitted signal $35 \mu$ s after the laser pulse. The emission spectra in the visible spectral range were corrected for the wavelength sensitivity of the detector. In a separate measurement, one ml of perfluoro-15-crown-5 (Exfluor Research Corporation) in a $4 \mathrm{~mm}$ NMR tube was saturated with oxygen at room temperature by bubbling with oxygen gas. This oxygen saturated solution was shock-frozen in the liquid nitrogen dewar $(77 \mathrm{~K})$ and luminescence experiments were performed as described above.

\section{Results and discussion}

The excitation of molecular oxygen by the $1064 \mathrm{~nm}$ laser pulse produces the following photophysical processes:

$$
\begin{gathered}
{\left[{ }^{3} \Sigma_{\mathrm{g}}{ }^{-}(v=0)\right]+h v[1064 \mathrm{~nm}] \rightarrow\left[{ }^{1} \Delta_{\mathrm{g}}(v=1)\right]} \\
{\left[{ }^{1} \Delta_{\mathrm{g}}(v=1)\right] \rightarrow\left[{ }^{1} \Delta_{\mathrm{g}}(v=0)\right]+\text { heat }} \\
{\left[{ }^{1} \Delta_{\mathrm{g}}(v=0)\right] \rightarrow\left[{ }^{3} \Sigma_{\mathrm{g}}{ }^{-}(v=0)\right]+h v[1270 \mathrm{~nm}]} \\
{\left[{ }^{1} \Delta_{\mathrm{g}}(v=0)\right] \rightarrow\left[{ }^{3} \Sigma_{\mathrm{g}}{ }^{-}(v=0)\right]+\text { heat }} \\
{\left[{ }^{1} \Delta_{\mathrm{g}}(v=0)\right]+\left[{ }^{1} \Delta_{\mathrm{g}}(v=0)\right] \rightarrow\left[{ }^{1} \Delta_{\mathrm{g}}(v=0)\right]_{2}} \\
{\left[{ }^{1} \Delta_{\mathrm{g}}(v=0)\right]_{2} \rightarrow 2\left[{ }^{3} \Sigma_{\mathrm{g}}{ }^{-}(v=0)\right]+h v[635 \mathrm{~nm}]} \\
{\left[\Delta_{\mathrm{g}}(v=0)\right]_{2} \rightarrow\left[{ }^{3} \Sigma_{\mathrm{g}}{ }^{-}(v=0)\right]+\left[{ }^{3} \Sigma_{\mathrm{g}}{ }^{-}(v=1)\right]+h v[704 \mathrm{~nm}]} \\
{\left[{ }^{1} \Delta_{\mathrm{g}}(v=0)\right]_{2} \rightarrow 2\left[{ }^{1} \Delta_{\mathrm{g}}(v=0)\right]+\text { heat }} \\
{\left[{ }^{1} \Delta_{\mathrm{g}}(v=0)\right]_{2} \rightarrow\left[{ }^{1} \Sigma_{\mathrm{g}}{ }^{+}(v=0)\right]+\left[{ }^{3} \Sigma_{\mathrm{g}}{ }^{-}(v=0)\right]+\text { heat }} \\
{\left[{ }^{1} \Sigma_{\mathrm{g}}{ }^{+}(v=0)\right] \rightarrow\left[{ }^{3} \Sigma^{-}{ }_{\mathrm{g}}(v=0)\right]+h v[765 \mathrm{~nm}]} \\
{\left[{ }^{1} \Sigma_{\mathrm{g}}{ }^{+}(v=0)\right] \rightarrow\left[{ }^{3} \Sigma_{\mathrm{g}}{ }^{-}(v=0)\right]+\text { heat }}
\end{gathered}
$$

Process (5) is the direct excitation to the vibronically excited state $\left[{ }^{1} \Delta_{\mathrm{g}}(v=1)\right]$ and its absorption coefficient is known to increase with oxygen concentration. ${ }^{3}$ Processes ((6), (8), (11), (12), (13) and (15)) are radiationless: decay, intersystem crossing and quenching events. Process (9) is the formation of the molecular pair excited states by collision. In this paper we will focus on radiative processes ((7), (10), (11) and (14)). We carried out spectral measurements on molecular oxygen in condensed phase and in a solution of fluorinated hydrocarbon. All samples were measured sequentially twice: first in the NIR region then in the visible.

\section{Emission measurement}

Fig. 2(A) shows the time resolved luminescence spectrum of the condensed phase of oxygen in the NIR region at $77 \mathrm{~K}$. The spectrum is assigned to the emission from the lowest excited state $\left[{ }^{1} \Delta_{\mathrm{g}}(v=0)\right]$ to ground state $\left[{ }^{3} \Sigma_{\mathrm{g}}{ }^{-}(v=0)\right]$ and is centered at $1270 \mathrm{~nm}$, (eqn (7)). The band intensity is directly proportional to \% oxygen concentrations and no emission was observed in the absence of oxygen $\left(100 \%\right.$ nitrogen). The emission from $\left[{ }^{1} \Delta_{\mathrm{g}}(v=0)\right]$ to the vibronic ground state $\left[{ }^{3} \Sigma_{\mathrm{g}}{ }^{-}(v=1)\right]$ observed at $1560 \mathrm{~nm}$ was not detected due to the lack of response above $1400 \mathrm{~nm}$ of the InGaAs photomultiplier model used. 


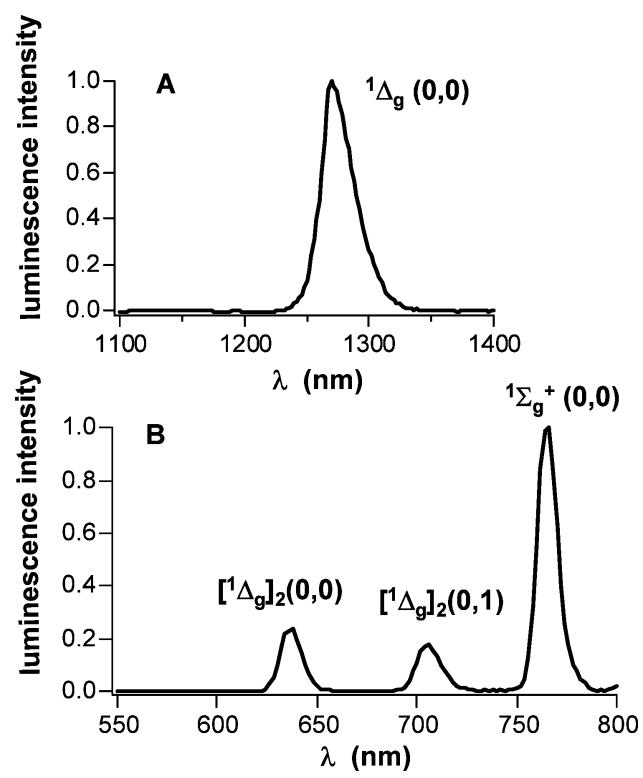

Fig. 2 (A) Time resolved phosphorescence spectrum $(30-80 \mu \mathrm{s})$ of condensed oxygen at $77 \mathrm{~K}$ excited at $1064 \mathrm{~nm}$ and detected in the NIR region. (B) The emission spectrum of condensed molecular oxygen excited at $1064 \mathrm{~nm}$ and detected in the visible region.

Fig. 2(B) shows the time resolved luminescence spectrum in the visible region. The spectrum has three bands, the $635 \mathrm{~nm}$ and $704 \mathrm{~nm}$ bands represent the simultaneous electronic transition of two molecules excited at $\left[{ }^{1} \Delta_{\mathrm{g}}(v=0)\right]$ (eqn (9)) emitting a single photon to ground state $\left[{ }^{3} \Sigma_{\mathrm{g}}{ }^{-}(v=0)\right]$. The two molecules emit at $635 \mathrm{~nm}$ if both molecules arrive to the ground state $\left[{ }^{3} \Sigma_{\mathrm{g}}{ }^{-}(v=\right.$ $0)$ ] simultaneously as in eqn (10). The two molecules emit at $704 \mathrm{~nm}$ if one molecule arrives to the first vibrational ground state $\left[{ }^{3} \Sigma_{\mathrm{g}}{ }^{-}(v=1)\right]$ as eqn (11). The separation between the two bands is $1543 \mathrm{~cm}^{-1}$ which is in good agreement with the ground electronic state vibrational frequency of $1556 \mathrm{~cm}^{-1}$. The emission of the two bands are mirror image to the absorption bands $\left[{ }^{1} \Delta_{\mathrm{g}}\right]_{2(0,0)}$ and $\left[{ }^{1} \Delta_{\mathrm{g}}\right]_{2(1,0)}$ shown in Fig. 1. We observed no emission from the combined simultaneous transition $\left[{ }^{1} \Sigma_{\mathrm{g}}{ }^{+}(v=0)\right]\left[{ }^{1} \Delta_{\mathrm{g}}(v=\right.$ $0)$ ] previously reported as weak emission band at $478 \mathrm{~nm} .{ }^{9}$ The band at $765 \mathrm{~nm}$ (Fig. 2(B)) is assigned to the emission from the single molecule $\left[{ }^{3} \Sigma_{\mathrm{g}}{ }^{+}(v=0)\right]$ electronic state generated by energy pooling from the $\left[{ }^{1} \Delta_{\mathrm{g}}\right]_{2}$ simultaneous transition as illustrated by eqn (13) and eqn (14). The relative intensity of the ${ }^{1} \Sigma_{\mathrm{g}}{ }^{+}$band is greater than the intensities of $\left[{ }^{1} \Delta_{\mathrm{g}}\right]_{2}$ bands, which is in contrast to the band ratio observed in the emission measurement from the chemiluminescence reaction ${ }^{9}$ The differences in the FranckCondon factors are not understood and similar discrepancies were observed in the singlet oxygen emission generated by electrical discharge of gaseous oxygen. ${ }^{10}$

\section{Lifetime measurement}

Fig. 3 shows luminescence decay traces measured at $1270 \mathrm{~nm}$, $765 \mathrm{~nm}, 636 \mathrm{~nm}$ and $704 \mathrm{~nm}$. The emission decay curves fitted well to pseudo-first order kinetics. The sharp spikes at the start of the decay curves are attributed to scatter of the laser light. Plot A is the decay curve of the $\left[{ }^{1} \Delta_{\mathrm{g}}\right]$ emission and has a lifetime of $46 \mu \mathrm{s}$. Plot B represents the formation of the $\left[{ }^{1} \Sigma_{\mathrm{g}}{ }^{+}\right]$state and is followed by the decay process. The emission intensity rise time
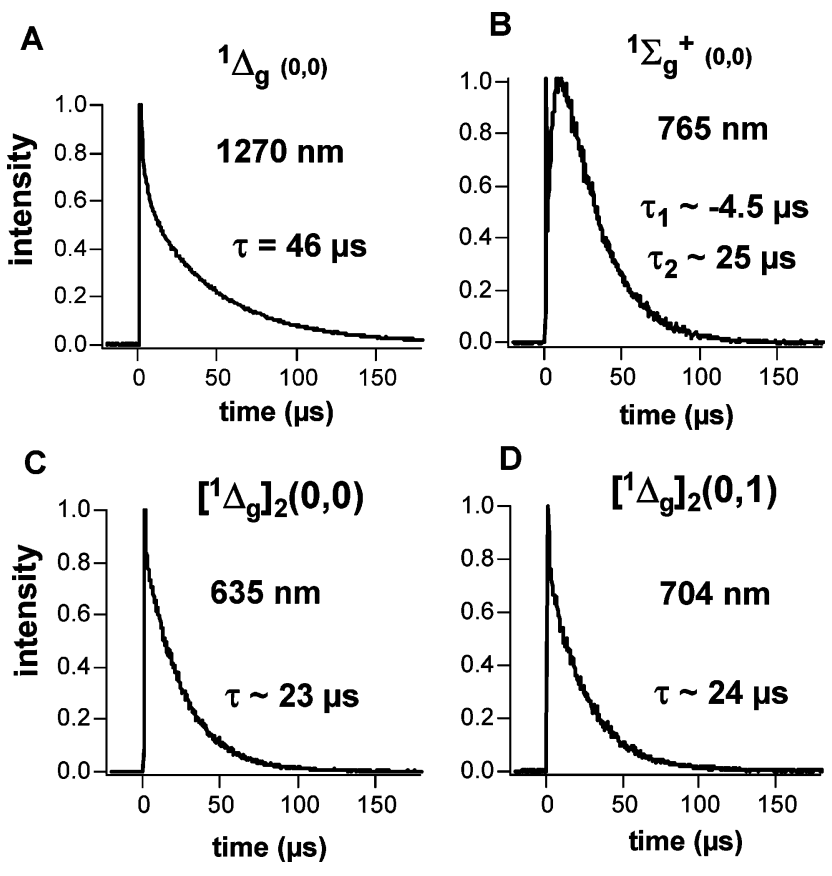

Fig. 3 Luminescence decay traces of condensed molecular oxygen at $77 \mathrm{~K}$ excited at $1064 \mathrm{~nm}$ and detected at (A) $1270 \mathrm{~nm}$, (B) $765 \mathrm{~nm}$, (C) $636 \mathrm{~nm}$, and (D) $704 \mathrm{~nm}$.

is measured as $4.5 \mu \mathrm{s}$ and the decay lifetime is $24 \mu \mathrm{s}$. Plots $\mathrm{C}$ and $\mathrm{D}$ are the decay curves of $\left[{ }^{1} \Delta_{\mathrm{g}}\right]_{2}$ emissions and both have lifetimes of $25 \mu$ s. Similar decay lifetimes were observed by Maier and coworkers for $\left[{ }^{1} \Delta_{\mathrm{g}}\right](43 \mu \mathrm{s})$ and $\left[{ }^{1} \Delta_{\mathrm{g}}\right]_{2}(22 \mu \mathrm{s})$ under similar conditions (excitation at $1064 \mathrm{~nm}$ of condensed oxygen at $77 \mathrm{~K}$ ). ${ }^{21}$ The decay lifetime of $\left[{ }^{1} \Delta_{\mathrm{g}}\right]_{2}$ is approximately half of the value for $\left[{ }^{1} \Delta_{\mathrm{g}}\right]$, which is in agreement with the studies of Kazakov and Schmidt of luminescence decay kinetics at room temperature in different solvents. ${ }^{22}$ These authors showed that the factor of two between the decay lifetimes of $\left[{ }^{1} \Delta_{\mathrm{g}}\right]$ and $\left[{ }^{1} \Delta_{\mathrm{g}}\right]_{2}$ is expected due to the fast equilibrium between 2 molecules of $\left[{ }^{1} \Delta_{\mathrm{g}}\right]$ and $\left[{ }^{1} \Delta_{\mathrm{g}}\right]_{2}$ in conjunction with the deactivation of $\left[{ }^{1} \Delta_{\mathrm{g}}\right]$ to $\left[{ }^{3} \Sigma_{\mathrm{g}}{ }^{-}\right]$.

Fig. 4 are the phosphorescence decay traces of the $\left[{ }^{1} \Delta_{\mathrm{g}}(v=\right.$ $0)$ ] emission detected at $1270 \mathrm{~nm}$ at three different oxygen concentrations at $77 \mathrm{~K}$. The lifetime $\tau_{1270 \mathrm{~nm}}$ decreases at higher $\%$

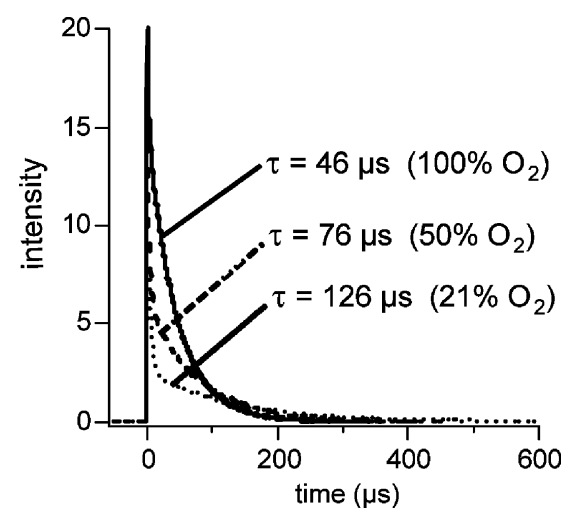

Fig. 4 Luminescence decay traces of condensed molecular oxygen excited at $1064 \mathrm{~nm}$ in three oxygen-nitrogen mixtures of different ratios detected at $1270 \mathrm{~nm}$ at $77 \mathrm{~K}$. 
oxygen as result of the quenching by $\left[{ }^{3} \Sigma_{\mathrm{g}}{ }^{-}\right]$. It should be noted that the oxygen concentration in the gas mixtures are not the same as in the condensed phase as the ratios are temperature dependent. The short lifetime of singlet oxygen in liquid oxygen of $46 \mu$ s is caused by self-quenching. At reduced oxygen concentration in liquid air, a longer lifetime of $126 \mu$ s was observed.

\section{Laser power dependence}

Using a set of neutral density filters we determined the emission intensity dependency on the laser power. Measurements on the $1270 \mathrm{~nm}, 765 \mathrm{~nm}$ and $635 \mathrm{~nm}$ emission bands are plotted in Fig. 5. The results clearly show that for $\left[{ }^{1} \Delta_{\mathrm{g}}\right]$ the emission intensity is linearly dependent on the laser power. This is in accord with a onephoton, one-molecule process. On the other hand the emission intensities for $\left[{ }^{1} \Delta_{\mathrm{g}}\right]_{2}$ and $\left[{ }^{1} \Sigma_{\mathrm{g}}{ }^{+}\right]$, exhibit a quadratic laser power dependence. Both transitions require two photons to be absorbed for each photon emitted.

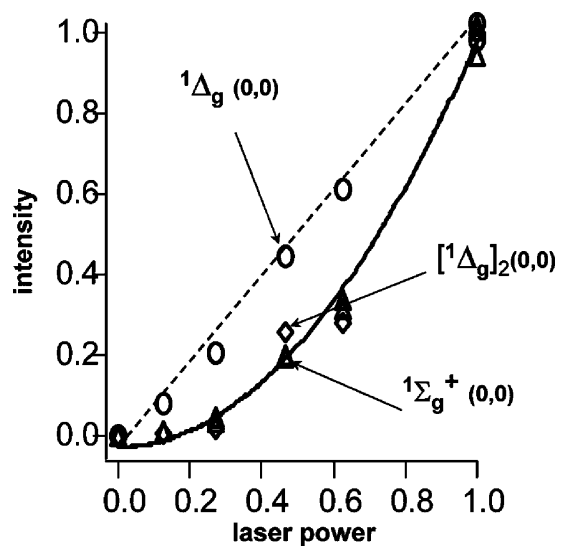

Fig. 5 Laser power dependencies on the emission intensity of ${ }^{1} \Delta_{g(0,0)}$, $2\left[{ }^{1} \Delta_{\mathrm{g}}\right]_{(0,0)}$, and ${ }^{1} \Sigma_{\mathrm{g}}{ }^{+}(0,0)$, , detected at $1270 \mathrm{~nm}, 636 \mathrm{~nm}$ and $765 \mathrm{~nm}$ respectively. $100 \%$ laser power corresponds to $\sim 100 \mathrm{~mJ}$ pulse $^{-1}$.

The NIR emission spectrum of shock frozen (77 K) oxygen saturated solutions of perfluoro-15-crown-5 is shown in Fig. 6. The spectrum exhibits a single band centered at $1270 \mathrm{~nm}$. The spectrum is similar to that of condensed oxygen (Fig. 2(A)). The insert of Fig. 6 compares the decay curves of oxygen saturated and argon saturated solutions both monitored at $1270 \mathrm{~nm}$. The oxygen saturated solution show a life time value of $141 \mu \mathrm{s}$, whereas the decay trace of the argon saturated solution has no measurable lifetime and follows the laser pulse. To test direct excitation of dissolved oxygen in a perfluorinated polymer film, samples of

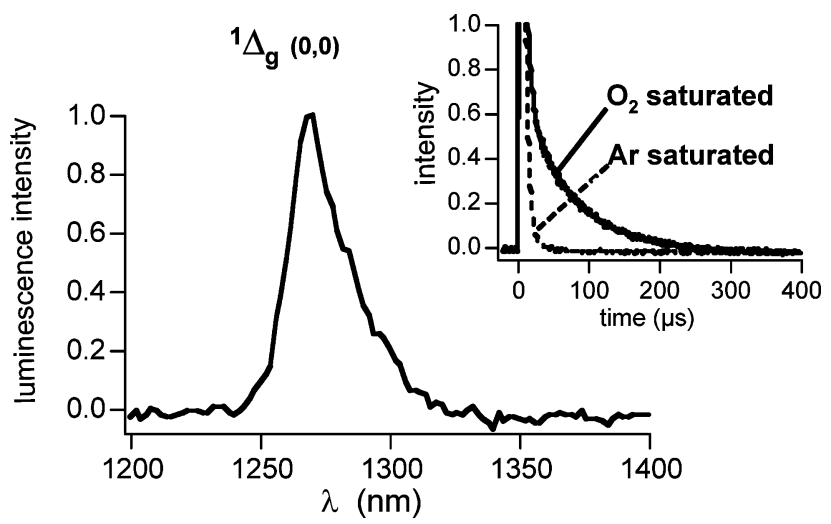

Fig. 6 Time resolved phosphorescence spectrum (30-80 $\mu$ s) of oxygen saturated perfluoro 15-crown-5 ether solution after excitation at $1064 \mathrm{~nm}$ at $77 \mathrm{~K}$. Insert: decay traces of oxygen (-) and argon (---) saturated perfluoro-15-crown-5 ether solutions monitored at $1270 \mathrm{~nm}$ following laser excitation $(1064 \mathrm{~nm})$ at $77 \mathrm{~K}$.

Teflon tape were equilibrium with $100 \%$ gaseous oxygen at $150 \mathrm{~K}$ and excited with laser pulses at $1064 \mathrm{~nm}$. A ${ }^{1} \Delta_{\mathrm{g}}$ emission life time of $530 \mu$ s was observed. The longer life time values observed in the samples containing oxygen in the saturated perfluorinated solvent and in the perfluorinated polymer are attributed to lower oxygen concentrations compared to the value measured in the condensed phase at $77 \mathrm{~K}$. All measured lifetimes values are listed in Table 1.

\section{Conclusions}

The results contained in this paper have implications for both fundamental and applied aspects of directly photo-excited oxygen luminescence. Recognizing the low quantum efficiency of the proposed process, the approach has certain advantages. (i) It requires no chemical reagents for measuring oxygen concentrations. (ii) Exciting and detecting in the NIR region has low ambient light interference and provides an opportunity for simultaneous measurement in the visible region.

In the area of basic research we will generate the ${ }^{1} \Sigma_{\mathrm{g}}{ }^{+}$state directly using known laser line at $760 \mathrm{~nm}$ of the condensed molecular oxygen. It should be noted that spectral measurements for the radiative transition from ${ }^{1} \Sigma_{\mathrm{g}}{ }^{+}$to ${ }^{3} \Sigma_{\mathrm{g}}{ }^{-}$or ${ }^{1} \Delta_{\mathrm{g}}$ were previously reported. ${ }^{23-25} \mathrm{We}$ will focus on investigating the following simultaneous transitions:

$$
\begin{gathered}
{\left[{ }^{1} \Sigma_{\mathrm{g}}{ }^{+}(v=0)\right]_{2} \rightarrow\left[{ }^{1} \Delta_{\mathrm{g}}(v=0)\right]+\left[{ }^{3} \Sigma_{\mathrm{g}}{ }^{-}(v=0)\right]+h v[548 \mathrm{~nm}]} \\
{\left[{ }^{1} \Sigma_{\mathrm{g}}{ }^{+}(v=0)\right]_{2} \rightarrow\left[{ }^{1} \Delta_{\mathrm{g}}(v=0)\right]_{2}+h v[1040 \mathrm{~nm}]}
\end{gathered}
$$

Table 1 Lifetimes of $\left[{ }^{1} \Delta_{\mathrm{g}}\right],\left[{ }^{1} \Sigma_{\mathrm{g}}{ }^{+}\right]$and $2\left[{ }^{1} \Delta_{\mathrm{g}}\right]$ by direct excitation

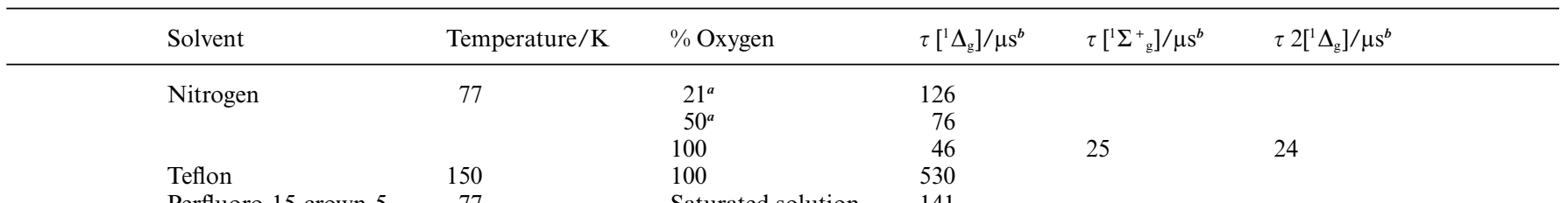

${ }^{a}$ Fraction of oxygen in oxygen-nitrogen gas mixtures, which were used to condense oxygen-nitrogen mixtures at $77 \mathrm{~K}$. The actual oxygen fraction in the condensed phase at $77 \mathrm{~K}$ might be different from the gas mixtures. ${ }^{b}$ Error limit $\sim 5 \%$. 
For initial application of direct excitation and detection of singlet oxygen we will focus on its potential use in cryogenic wind tunnel testing. ${ }^{26,27}$ We plan to develop a novel method for obtaining quantitative non-intrusive mapping of the 2-D oxygen/pressure at many points. The image intensity of the $1270 \mathrm{~nm}$ luminescence should generate a map whose intensities are proportional to the oxygen concentration which is directly proportional to pressure.

\section{Acknowledgements}

The authors at the University of Washington thank the Air Force Office of Scientific Research STTR AF04-T001, Innovative Scientific Solutions Inc. and the National Science Foundation (Grant 0517782) for financial support. The authors at Columbia thank the National Science Foundation for financial support under Grant CHE 04-15516.

\section{References}

1 R. S. Mulliken, Interpretation of the atmospheric oxygen bands; electronic levels of the oxygen molecule, Nature, 1928, 122, 505.

2 R. S. Mulliken, Interpretation of band spectra. III. Electron, quantum numbers and states of molecules and their atoms, Rev. Mod. Phys., $1932,4,1-86$

3 C. Schweitzer and R. Schmidt, Physical mechanisms of generation and deactivation of singlet oxygen, Chem. Rev., 2003, 103, 1685-1757.

4 G. W. Robinson, Intensity enhancement of forbidden electronic transitions by weak intermolecular interactions, J. Chem. Phys., 1967, 46, $572-585$.

5 V. G. Krishna, Simultaneous and induced electronic transitions in oxygen. I., J. Chem. Phys., 1969, 50, 792-799.

6 C. Long and D. R. Kearns, Selection-rules for intermolecular enhancement of spin forbidden transitions in molecular-oxygen, J. Chem. Phys., 1973, 59, 5729-5736.

7 P. R. Ogilby, Solvent effects on the radiative transitions of singlet oxygen, Acc. Chem. Res., 1999, 32, 512-519.

8 E. W. Ellis and H. O. Kneser, Kombinationsbeziehungen im Absorptionsspektrum des flüssigen Sauerstoffs, Z. Phys., 1933, 86, 583-591.

9 A. U. Khan and M. Kasha, Chemiluminescence arising from simultaneous transitions in pairs of singlet oxygen molecules, J. Am. Chem. Soc., 1970, 92, 3293-3300.

10 S. J. Arnold, E. A. Ogryzlo and H. Witzke, Some new emission bands of molecular oxygen, J. Chem. Phys., 1964, 40, 1769-1770.

11 Singlet Molecular Oxygen, ed. M. S. Chadha, Bhabha Atomic Research Centre, Bombay, India, 1975.
12 A. P. Schaap, Singlet Molecular Oxygen, Dowden, Hutchinson \& Ross, Stroudsburg, PA, 1976.

13 A. Frimer, Singlet Oxygen, CRC Press, Boca Raton, FL, 1985.

14 R. W. Redmond and I. E. Kochevar, Symposium-in-print: singlet oxygen, Photochem. Photobiol., 2006, 82, 1178-1186.

15 R. Schmidt, C. Tanielian, R. Dunsbach and C. Wolff, Phenalenone, a universal reference compound for the determination of quantum yields of singlet oxygen $\mathrm{O}_{2}{ }^{1} \Delta_{\mathrm{g}}$ ) sensitization, J. Photochem. Photobiol., A, 1994, 79, 11-17.

16 I. Rosenthal, Phthalocyanines as photodynamic sensitizers, Photochem. Photobiol., 1991, 53, 859-870.

17 G. Khalil, A. Chang, M. Gouterman, J. B. Callis, L. R. Dalton, N. J. Turro and S. Jockusch, Oxygen pressure measurement using singlet oxygen emission, Rev. Sci. Instrum., 2005, 76, 054101-1-054101-8.

18 S. Jockusch, J. Sivaguru, N. J. Turro and V. Ramamurthy, Direct measurement of the singlet oxygen lifetime in zeolites by near-IR phosphorescence, Photochem. Photobiol. Sci., 2005, 4, 403-405.

19 L. Skuja and B. Güttler, Detection of interstitial oxygen molecules in $\mathrm{SiO}_{2}$ glass by direct photoexcitation of the infrared luminescence of singlet $\mathrm{O}_{2}$, Phys. Rev. Lett., 1996, 77, 2093-2096.

20 A. A. Krasnovsky, Jr., Ya. V. Roumbal, A. V. Ivanov and R. V. Ambartzumian, Solvent dependence of the steady-state rate of ${ }^{1} \mathrm{O}_{2}$ generation upon excitation of dissolved oxygen by cw $1267 \mathrm{~nm}$ laser radiation in air-saturated solutions: Estimates of the absorbance and molar absorption coefficients of oxygen at the excitation wavelength, Chem. Phys. Lett., 2006, 430, 260-264.

$21 \mathrm{E}$. Wild, H. Klingshirn and M. Maier, Relaxation of the ${ }^{1} \Delta_{\mathrm{g}}$ state in pure liquid oxygen and in liquid mixtures of ${ }^{16} \mathrm{O}_{2}{ }^{18} \mathrm{O}_{2}, J$. Photochem., 1984, 25, 131-143.

22 D. V. Kazakov and R. Schmidt, On the effect of 1,4-diazabicyclo[2.2.2] octane on the singlet-oxygen dimol emission: Photosensitized generation of $\left({ }^{1} \mathrm{O}_{2}\right)_{2}$, J. Phys. Chem. A, 2007, 111, 4274-4279.

23 F. Noxon, Observation of the b-a transition in $\mathrm{O}_{2}$, Can. J. Phys., 1961, 39, 1110-1119.

24 T. Keszthelyi, D. Weldon, T. N. Anderson, T. D. Poulssen, K. V. Mikkelsen and P.R. Ogilby, Radiative transitions of singlet oxygen: New tools, new techniques and new interpretations, Photochem. Photobiol., 1999, 70, 531-539.

25 R. Schmidt and M. Bodesheim, Time-resolved measurement of $\mathrm{O}_{2}\left({ }^{3} \Sigma_{\mathrm{g}}{ }^{+}\right)$in solution - Phosphorescence from an upper excited-state, J. Phys. Chem., 1994, 98, 2874-2876.

26 K. Asai, H. Kanda, C. T. Cunningham, R. Erausquin and J. P. Sullivan, Surface pressure measurement in a cryogenic wind tunnel by using luminescent coating, ICIASF '97 Record, Pacific Grove, CA, 1997, pp. $105-114$.

27 A. N. Watkins, W. K. Goad, C. J. Obara, D. R. Sprinkle, R. L. Campbell, M. B. Carter, O. C. Pendergraft, J. H. Bell, J. L. Ingram, D. M. Oglesby, P. J. Underwood and L. R. Humber, Flow visualization at cryogenic conditions using a modified pressure sensitive paint approach, AIAA Paper AIAA, 2005, 2005-456. 